\title{
Attributing the Human Disease Burden of Foodborne Infections to Specific Sources
}

Pires, Sara Monteiro; Evers, Eric E.; Van Pely, Wilfrid; Ayers, Tracy; Scallan, Elain; Angulo, Frederick J.; Havelaar, Arie; Hald, Tine

\author{
Published in: \\ Foodborne Pathogens and Disease
}

Link to article, DOI:

10.1089/fpd.2008.0208

Publication date:

2009

Link back to DTU Orbit

Citation (APA):

Pires, S. M., Evers, E. E., Van Pely, W., Ayers, T., Scallan, E., Angulo, F. J., Havelaar, A., \& Hald, T. (2009). Attributing the Human Disease Burden of Foodborne Infections to Specific Sources. Foodborne Pathogens and Disease, 6(4), 417-424. https://doi.org/10.1089/fpd.2008.0208

\section{General rights}

Copyright and moral rights for the publications made accessible in the public portal are retained by the authors and/or other copyright owners and it is a condition of accessing publications that users recognise and abide by the legal requirements associated with these rights.

- Users may download and print one copy of any publication from the public portal for the purpose of private study or research.

- You may not further distribute the material or use it for any profit-making activity or commercial gain

- You may freely distribute the URL identifying the publication in the public portal 


\title{
Review
}

\section{Attributing the Human Disease Burden of Foodborne Infections to Specific Sources}

\author{
Sara M. Pires, ${ }^{1}$ Eric G. Evers, ${ }^{2}$ Wilfrid van Pelt, ${ }^{3}$ Tracy Ayers, ${ }^{4}$ Elaine Scallan, ${ }^{4}$ \\ Frederick J. Angulo, ${ }^{4}$ Arie Havelaar, ${ }^{2,5}$ Tine Hald, ${ }^{1}$ and the Med-Vet-Net Workpackage 28 Working Group ${ }^{6}$
}

\begin{abstract}
Foodborne diseases are an important cause of human illness worldwide. Humans acquire these infections from a variety of sources and routes of transmission. Many efforts have been made in the last decades to prevent and control foodborne diseases, particularly foodborne zoonoses. However, information on the impact of these interventions is limited. To identify and prioritize successful food safety interventions, it is important to attribute the burden of human illness to the specific sources. Defining scientific concepts and harmonizing terminology for "source attribution" is essential for understanding and improving attribution methodologies and for sharing knowledge within the scientific community. We propose harmonized nomenclature, and describe the various approaches for human illness source attribution and their usefulness to address specific public health questions.
\end{abstract}

\section{Introduction}

$\mathbf{P}$ ATHOGENS COMMONLY TRANSMITTED to humans through foods are responsible for a high burden of human illness and death worldwide. The World Health Organization (WHO) estimates that 1.8 million children die each year from diarrhea, and much of the childhood diarrhea is caused by pathogens that are commonly acquired from contaminated food or water. Furthermore, even in developed countries up to one third of the population each year has an infection from a pathogen commonly transmitted through foods (WHO, 2005). Humans acquire these infections through a number of routes, including eating contaminated food, contact with live animals, and contact with a contaminated environment. Foodborne transmission is recognized as being responsible for a major proportion of these infections, and foodborne diseases may involve many different food sources and commodities. Several countries have implemented intervention programs during the last decades to prevent and control foodborne diseases, particularly foodborne zoonoses (Wegener et al., 2003; EFSA, 2006). However, precise measurement of the public health impact of such interventions has been difficult, in part because information on the attribution of the burden of foodborne diseases to specific sources is often insufficient. To prioritize appropriate food safety interventions, it is crucial to attribute the human disease burden of each foodborne infection to specific sources (FAO/WHO, 2006).

A variety of general methods to attribute one or more foodborne diseases to specific sources has been developed, including microbiological approaches, epidemiological

\footnotetext{
${ }^{1}$ National Food Institute, Technical University of Denmark, Lyngby, Denmark.

${ }^{2}$ Laboratory of Zoonoses and Environmental Microbiology, National Institute for Public Health and the Environment, Bilthoven, The Netherlands.

${ }^{3}$ Epidemiology and Surveillance Unit, National Institute for Public Health and the Environment, Bilthoven, The Netherlands.

${ }^{4}$ Enteric Disease Epidemiology Branch, Division of Foodborne, Bacterial, and Mycotic Diseases, National Center for Zoonotic, Vectorborne, and Enteric Diseases, Centers for Disease Control and Prevention, Atlanta, Georgia.

${ }^{5}$ Institute for Risk Assessment Sciences, Utrecht University, Utrecht, The Netherlands.

${ }^{6}$ In addition to the authors: Andreas Schroeter (Federal Institute for Risk Assessment [BfR], Germany), Anne Brisabois (French Food Safety Agency [AFSSA]), Anne Thebault (AFSSA), Annemarie Käsbohrer (BfR), Carl Schroeder (United States Department of Agriculture [USDA]), Christina Frank (Robert Koch Institut [RKI], Germany), Chuanfa Guo (USDA-Food Safety and Inspection Service [FSIS]), Danilo Lo Fo Wong (National Food Institute [NFI], Technical University of Denmark), Dörte Döpfer (Wageningen University, The Netherlands), Emma Snary (Veterinary Laboratories Agency [VLA], UK), Gordon Nichols (Health Protection Agency, UK), Heidi Spitznagel (BfR), Helene Wahlström (National Veterinary Institute, Sweden), Julie David (AFSSA), Katarzyna Pancer (National Public Health Institute, Poland), Klaus Stark (RKI), Lars Plym Forshell (National Food Administration, Sweden), Padraig Nally (VLA), Pascal Sanders (AFSSA), and Petra Hiller (BfR).
} 
approaches, intervention studies, and expert elicitation approaches. Each of these general methods presents advantages and limitations, and the usefulness of each depends on the public health questions being addressed (Batz et al., 2005). Several groups are using attribution methods, but these often have different nomenclature and food categorization schemes. Defining scientific concepts and harmonizing terminology are essential for understanding and improving attribution methodologies and sharing knowledge across the scientific community. In this paper, we propose harmonized nomenclature; describe different approaches to human illness attribution; and discuss the advantages, limitations, and applicability of each approach in answering different questions along the farm-to-consumption continuum, while emphasizing that the choice of the attribution method will depend on the pathogen and the public health question being addressed.

\section{Definitions}

Human illness "source attribution" may be defined as the partitioning of the human disease burden of one or more foodborne infections to specific sources, where the term source includes animal reservoirs and vehicles (e.g., foods). The human disease burden can be measured by the number of laboratory-confirmed (reported) infections or by the estimated total number of infections. To enable comparisons and to account for morbidity and mortality, the human disease burden can be expressed as disability adjusted life years (DALYs).

Attribution of human illness to specific sources requires categorization of the sources. Harmonization of the categorization scheme is needed for comparisons and integration of results from various models and approaches. Such a system should be hierarchical, while accommodating different levels of detail required for different purposes. The categorization scheme should fit with food consumption databases and be internationally standardized.

\section{General Approaches for Source Attribution}

\section{Microbiological approaches}

One general method for attribution of the human disease burden of foodborne infections to specific sources is "microbiological approaches." Microbiological approaches for source attribution include the microbial subtyping approach and the comparative exposure assessment approach. Both approaches involve isolation of the pathogen from the various sources and from ill humans. The microbial subtyping approach requires a representative distribution of the subtypes of the pathogen in the different sources and humans, but does not depend on estimates of the prevalence of the subtypes in each source. The comparative exposure assessment approach requires estimates of the prevalence and concentration of the pathogen in each of the sources of exposure.

Microbial subtyping approach. The microbial subtyping approach involves characterization of isolates of a specific pathogen by phenotypic and/or genotypic subtyping methods (e.g., serotyping, phage typing, antimicrobial susceptibility testing, pulsed-field gel electrophoresis, sequence-based subtyping). The principle is to compare the subtypes of isolates from different sources (e.g., animals, food) with those isolated from humans. The microbial subtyping approach is enabled by the identification of strong associations between some of the dominant subtypes and a specific reservoir or source, providing a heterogeneous distribution of subtypes among the sources. As a first step, subtypes isolated exclusively or almost exclusively from one source are regarded as "indicator subtypes," and the human infections caused by each indicator subtype are assigned (attributed) to that specific source. The relationship between the relative occurrence (i.e., proportion of positive samples or positive isolates) of each indicator subtype in the source and the incidence of human infections caused by that indicator subtype is then determined. Finally, human infections caused by subtypes found in several sources are assigned to specific sources proportional to the occurrence of the indicator subtypes. The application of this approach assumes that the distribution of subtypes in the collection of isolates in each source used in the attribution exercise is similar to the true distribution of subtypes in each source. Because the microbial subtyping approach utilizes a collection of temporally and spatially related isolates from various sources, it is facilitated by an integrated foodborne disease surveillance program that is focused on the collection of isolates from the major food animal reservoirs of foodborne diseases.

There have been several applications of the microbial subtyping approach for Salmonella source attribution (e.g., Van Pelt et al., 1999; Sarwari et al., 2001). The most advanced application of the microbial subtyping approach for Salmonella was developed in Denmark (Hald et al., 2004). Using data from the integrated Danish Salmonella surveillance program, a mathematical model was developed to quantify the contribution of each of the major food animal sources to human Salmonella infections. The "Danish Salmonella source account" model attributes domestically acquired laboratory-confirmed human Salmonella infections caused by different Salmonella subtypes (serotypes and phage types) as a function of the prevalence of these subtypes in animal and food sources and the amount of each food source consumed, using a Bayesian framework with Markov chain Monte Carlo simulation (Gilks et al., 1996). This microbial subtyping approach has proved to be a valuable tool in focusing food safety interventions to the appropriate animal reservoir in Denmark and provides an example of potential synergy between quantitative risk assessment and public health surveillance (Hald et al., 2004).

Another example of the microbial subtyping approach is the use of multilocus sequence typing (MLST) of Campylobacter jejuni isolates from foods and humans, being applied in the United Kingdom (Dingle et al., 2002) and New Zealand (French, 2007). In this microbial subtyping approach, MLST is used to identify lineages in bacterial populations by indexing the variation present in seven housekeeping genes located in various parts of the chromosome (Dingle et al., 2001). With the development of novel analysis tools such as the ClonalFrame algorithm (Didelot and Falush, 2007), MLST has been used to identify clonal complexes associated with different isolation sources that, in some instances, correspond to different host species. As an increasing number of isolates, including isolates from various sources, are added to the MLST database (accessible on the internet), there will be increased precision in the attribution of human infections to host sources (McCarthy et al., 2007). Recently, MLST data were utilized to attribute the sources of human C. jejuni infections in New Zealand using two microbial subtyping models, the ClonalFrame algorithm 
and the Danish Salmonella source account; both models gave similar results (French, 2007).

When the microbial subtyping approach is applied at the point of production, it quantifies the contribution of the most important reservoirs of the specific pathogen, assuming isolates are available from those reservoirs. Because pathogens can be transmitted through a variety of sources, interventions that control the pathogen at the reservoir level, before the dissemination of the pathogen through numerous transmission pathways, should result in important declines in human infections.

A limitation is that the microbial subtyping approach is restricted to pathogens that are heterogeneously distributed among the sources. Furthermore, the microbial subtyping approach will only attribute illness to those sources from which isolates are available. The microbial subtyping approach is also data intensive and requires a sufficiently large and representative sample from each source. Independent of the typing method used, the application of strain typing to make inferences on the sources of human infections must be done with appreciation of the strengths and limitations of typing methods (Olsen et al., 1993; Tenover et al., 1997). Clonality is defined as the high probability that two isolates that are identical by typing are related to each other, and the confidence in this probability becomes greater when more than one typing method is applied (Olsen et al., 1993).

Comparative exposure assessment approach. The principle of the comparative exposure assessment approach is to determine the relative importance of the known transmission routes by estimating the human exposure to that pathogen via each route. The comparative exposure assessment approach requires, for each known transmission route, information on the prevalence and dose of the pathogen in the source, the changes of the prevalence and quantity of the pathogen throughout the transmission chain, and the frequency at which humans are exposed by that route. These data provide an estimate of the exposure dose for each transmission route. The exposure doses are compared and the human disease burden (e.g., the observed laboratory-confirmed infections or estimated total number of infections) caused by the specific pathogen is partitioned to each of the various transmission routes, proportionally to the size of the exposure dose. The estimates of exposure dose for each transmission route can be combined with a dose-response model to predict the number of infections from each route, similar to what is done in traditional microbial risk assessments. The comparative exposure assessment approach for source attribution differs from traditional risk assessment in its objective and level of detail. A risk assessment typically aims at describing the complex dynamics of a pathogen in a single food commodity during food processing, and predicting the relative public-health effect of different interventions strategies-alone and in combination. In contrast, the comparative exposure assessment aims at partitioning the observed (or predicted) human disease burden to all known transmission routes, including various foods, direct contact with live animals, and environmental exposures. For this purpose, the various transmission routes are modeled in a more simplified and less detailed way that represents only the main steps in the transmission pathway.

A comparative exposure assessment approach was used to attribute human Campylobacter infections to various trans- mission routes in the Netherlands (Evers et al., 2008). Human exposure across the major transmission routes was estimated in a stochastic model as the mean dose of Campylobacter ingested per person per day, averaged over the entire Dutch population. Thirty-one transmission routes related to ingestion of food and direct contact with animals and water were investigated. Another example of this approach was developed to attribute human Campylobacter infections in New Zealand, where a model was used to explore the relative importance of four of the most commonly identified routes of exposure: food (poultry and red meat), drinking water, freshwater swimming, and occupational contact (livestock). The model estimates the mean daily dose to which an average individual is exposed to from each route, and, by comparing the relative exposure doses, human Campylobacter infections were attributed to the sources (McBride et al., 2005).

The strength of the comparative exposure assessment approach is that it attributes human illness to sources of exposure, taking into account the different transmission routes from the same reservoir, e.g., estimating the role of dairy products and beef from the cattle reservoir. However, this approach is often limited by a lack of sufficient data (e.g., comparable prevalence data or data on exposure through different routes), which results in large uncertainties around the estimates.

\section{Epidemiological approaches}

A second general method for attribution of the human disease burden of foodborne diseases to specific sources is "epidemiological approaches." Epidemiological approaches for source attribution usually involve interviews of patients to elicit the patient's recall of foods consumed or other exposures before illness began. Some patient interviews are undertaken as a routine activity under the existing public health surveillance infrastructure. Additional studies may be performed to determine factors associated with apparently sporadic infections or in association with investigations of outbreaks of human infections. An outbreak is defined as the occurrence of two or more cases of a similar illness resulting from the exposure to a common source (Olsen et al., 2000), while sporadic cases represent cases that have not been associated with known outbreaks (Engberg, 2006). In some circumstances, cases classified as sporadic may belong to undetected outbreaks. Identification of possible sources of apparently sporadic infections and outbreaks may be undertaken using analytical epidemiological studies, which involve interviewing persons who are (or will become) ill and persons who are not (or will not become) ill, or case-series studies, which involve interviewing only individuals who are ill.

Studies of sporadic infections. Several types of studies have been performed to identify possible sources of apparently sporadic human infections. Case-control studies are the most commonly used analytical epidemiological studies for identifying possible exposures associated with sporadic infections. To allow sufficient enrollment of patients, casecontrol studies of sporadic infections are often conducted over an extended period of time and commonly use public health surveillance to ascertain culture-confirmed cases. Typically, selected case-patients and a corresponding group of asymptomatic, and therefore assumed to be uninfected, individuals 
(controls) are interviewed, and the relative role of exposures is estimated by comparing the frequency of exposures among cases and controls. When infections are associated with an exposure, the proportion of cases attributed to the exposure can be calculated and is defined epidemiologically as the "population attributable fraction" (PAF) (Clayton and Hills, 1993). The PAFs can be used to partition the human disease burden to specific sources (Stafford et al., 2008).

Numerous case-control studies of sporadic infections of diseases commonly transmitted through food, including zoonotic diseases, have been published. Some of these studies calculate only measures of association, e.g., Salmonella Enteritidis infections in Denmark (Mølbak and Neimann, 2002) and Campylobacter infections in Denmark (Wingstrand et al., 2006), while others estimate the PAF, e.g., Shiga toxin-producing Escherichia coli $\mathrm{O} 157$ infections in the United States (Voetsch et al., 2007). Case-control studies, when combined with data on the human health burden of the disease under study, can also be utilized to attribute that burden to specific sources. A good example is the recently published study for sporadic Campylobacter infections in Australia (Stafford et al., 2008), where the authors determine the PAF for various exposures, and combine the PAF estimates with estimates of the burden of Campylobacter to partition the burden to specific sources.

Case-control studies are a valuable tool to identify potential risk factors for human infections, including sources and predisposing, behavioral or seasonal factors (Engberg, 2006). Moreover, in addition to individual case-control studies, a systematic review of published case-control studies of sporadic infections of a given pathogen can provide an overview of the relevant exposures and risk factors for that infection, and a summary of the estimated population attributable fractions for each exposure. An overall population attributable fraction derived from a meta-analysis or weighted summary of several case-control studies of a certain pathogen can be combined with estimates of the burden of illness caused by that pathogen to estimate the burden of illness attributed to each exposure.

A limitation of epidemiological approaches, particularly case-control studies, is that cases that are not associated with a recognized outbreak reflect a mixture of possible sources of exposure, and it may be difficult to distinguish between these exposures. Another limitation is that the statistical power to determine the importance of common exposures often requires enrollment of many participants. Furthermore, a limitation of case-control studies is the lack of accuracy in the recall of exposures by participants (ill and well participants). This misclassification of exposures commonly leads to an underestimation of the burden of illness attributed to specific exposures (Stafford et al., 2008).

Cohort studies, another type of analytical epidemiological study, are used less often for sporadic infections, since they usually require interviewing more persons than is practical, most of whom are not infected. Examples of cohort studies performed to determine the overall disease burden attributable to specific pathogens include the study of sporadic infections by de Wit et al. (2002) and the Infectious Intestinal Disease study in the United Kingdom (Wheeler et al., 1999).

Case-series studies of sporadic infections are commonly conducted, particularly for uncommon diseases that have a well-recognized source of infection, to which persons without the infection are infrequently exposed. Examples of case series studies of sporadic infections and the common source for that disease include botulism associated with home-canned foods (Sobel et al., 2004), Vibrio vulnificus and oysters (Shapiro et al., 1998), and Salmonella Typhi infections and foreign travel in the United States (Ackers et al., 2000). When an exposure is uncommon in the general population, case series studies of a sporadic infection can be used to determine the frequency of that exposure among the cases, and that frequency can be considered against the proportion of the burden of illness caused by that disease that is attributed to that specific exposure.

Analysis of data from outbreak investigations. Another epidemiological approach for source attribution involves conducting an analysis of the information available from outbreak investigations. An investigation of an individual outbreak may involve both microbiological and epidemiological data; the epidemiological data may be derived from interviewing only infected persons (i.e., a case-series) or from interviewing both infected and noninfected persons. Typically, the microbiological data in an individual outbreak investigation are used to generate hypotheses about the source of the outbreak or to support the results of an epidemiological investigation. Many outbreak investigations are successful in identifying the specific source for the human infections. By conducting an analysis of data from outbreak investigations, the most common food vehicles involved in outbreaks can be identified. A simple analysis or summary of outbreak investigations is useful for attributing illnesses to foods, but often the implicated food item in an individual outbreak is a "complex" food, containing several food items, many of which could be the specific source of the infection. Several methods have been used to include the information of complex foods involved in outbreaks when conducting an analysis of data from outbreak investigations to attribute human illness to sources.

An example of an analysis of data from outbreak investigations for source attribution was developed in the United Kingdom. This analysis used data from individual outbreak investigations reported through national surveillance and population-based studies to estimate the number of human foodborne infections in England and Wales associated with specific food sources (Adak et al., 2005). In this method, individual outbreak investigations that implicated complex foods were not included in the analysis. An alternative method for conducting an analysis of data from outbreak investigations is being developed in the United States. In this method, food items implicated in foodborne outbreaks reported to the Centers for Disease Control and Prevention via the electronic Foodborne Outbreak Reporting System are used to attribute illnesses to specific foods. Food items are categorized into a hierarchical scheme, according to their ingredients. Foods that contain ingredients that are members of a single commodity are considered "simple foods," while foods that contain ingredients that are members of multiple commodities are considered "complex foods." As an example, ground beef is a simple food, whereas meat pie is a complex food. Each implicated food is assigned to one or more mutually exclusive food commodities, according to its ingredients. For outbreaks that have implicated a simple food item, all illnesses are attributed to that single commodity. For outbreaks that have implicated a complex food item, illnesses are partitioned to each commodity in the complex food according to the proportion of illnesses attributed to each of those commodities in outbreaks caused by simple foods. As a result, illnesses in an outbreak due to a 
complex food item are attributed to a commodity in the implicated complex food, only if that commodity has been implicated in at least one outbreak due to a simple food. The number of illnesses attributed to each commodity are then summed and used to determine the percentage of disease attributed to each commodity. To estimate the attribution of all foodborne illness in the United States (and not just illnesses involved in reported outbreaks), the percentages of illness for each disease attributed to each commodity were weighted by the estimated annual burden of illness for each pathogen.

A limitation of an analysis of data from individual outbreak investigations is that many outbreaks may not be detected, investigated, or reported. In many cases, a pathogen is not identified, the source is not elucidated, or both. Also, the quality of evidence varies and classification schemes for the data are not consistently used. Large outbreaks, outbreaks associated with point sources, outbreaks that have short incubation periods, and outbreaks that cause serious illness are more likely to be detected, investigated, and reported. As a consequence, the burden of illness in the population attributed to some food sources may be underestimated, which will likely vary by pathogen (Batz et al., 2005). Another limitation is that categorization of foods may differ from one analysis to another. A third limitation of an analysis of data from individual outbreak investigations is that foodborne illnesses included in data from outbreak investigations may not be representative of all foodborne illnesses. Finally, certain food vehicles are more likely to be associated with reported outbreaks than others, which can lead to an overestimation of the proportion of human illnesses attributed to a specific food.

An advantage of data from outbreak investigations is that clear documentation that a specific pathogen was transmitted to humans via a specific food item can be available. Furthermore, an analysis of data from outbreak investigations to attribute foodborne illness to specific sources may sometimes include information on the point of contamination at the farm to consumption chain, particularly when individual outbreak investigations have complete trace back information available. Another advantage is that a wide variety of food vehicles are represented, including less frequently identified food items (e.g., almonds or sprouts). Finally, an analysis of data from outbreak investigations may in some countries or regions be the most readily available source of information for human illness attribution purposes.

\section{Intervention studies}

A third general method to attribute the human disease burden of one or more foodborne infections to specific sources is the use of intervention studies. Intervention studies can provide compelling evidence of the burden of illness attributed to a specific source, particularly if the intervention is conducted in a randomized, double-blinded design. Intervention studies have proven useful, for example in developed countries in determining the population disease burden from a particular exposure (e.g., drinking water), and in developing countries in determining the burden of illness from a variety of hygienerelated factors. Intervention studies can be designed as smallscale (e.g., at farm level) or larger-scale (e.g., interventions at a national level) studies to control a certain foodborne disease. Examples of intervention studies include the experiences of reducing the burden of human campylobacteriosis from poultry meat in Iceland (Stern et al., 2003) and human salmonellosis in Denmark (Wegener et al., 2003).

Accidental interventions, where a decrease or increase in human disease incidence can be observed due to a change in exposure or behavior of the population at risk (e.g., sudden changes in consumption of certain food items) may occur. Examples include the sudden decline in beef consumption due to bovine spongiform encephalopathy in the United Kingdom, and declines in chicken consumption due to outbreaks of avian influenza in Europe. By analyzing changes in consumption and numbers of reported cases of human illness, it is possible to estimate the number of human cases attributable to specific sources. For example, in June 1999, the use of dioxin-contaminated chicken feed in Belgium resulted in the withdrawal of chicken meat and eggs from the market (Vellinga and Van Loock, 2002). A decrease in reported human Campylobacter infections also occurred in Belgium, beginning in June 1999. Public health surveillance data from preceding years (1994 to 1998) were used to predict the number of human Campylobacter infections expected in 1999. The number of reported cases in 1999 was substantially lower (40\%) than the expected, suggesting that $40 \%$ of reported human Campylobacter infections in Belgium could be attributed to chicken (Velling and Van Lock, 2002).

\section{Expert elicitation approaches}

A fourth general method to attribute the human disease burden of foodborne diseases to specific sources is "expert elicitation approaches." Expert elicitations are commonly used to address data gaps. Several studies have used expert elicitations to estimate the proportion of human infections that are foodborne, which can be viewed as a first step in source attribution (e.g., Mead et al., 1999; Van Duynhoven et al., 2002; Hoffmann et al., 2007).

One of the approaches used for expert elicitation is the generalized framework for expert judgment studies from heterogeneous expert panels based on the Group Delphi method (Henson, 1997), which has been adapted for source attribution of foodborne illness in New Zealand (Lake et al., 2006). The process involved selection and invitation of experts, distribution of summary sheets for each food-hazard combination, a group meeting and completion of a questionnaire (without discussion). The results of the questionnaires were then aggregated by Monte Carlo simulation of pert distributions with equal weighting of each participant and reported back to the group. A period of discussion followed and the estimates for the questionnaire were then repeated by each expert and re-aggregated. A final report was prepared and sent to each participant with the possibility of further comment. The discussion period was considered valuable in generating consensus and exchanging information (Lake et al., 2006). Another example of the use of a systematic expert elicitation approach for source attribution is the study performed to estimate the fraction of human cases of enterically transmitted illness by five major pathways (food, environment, direct animal contact, human-human transmission, and travel) and by 11 groups within the food pathway. In this study, 16 food safety experts were asked to provide their estimates of the most likely range for each of the parameters, and joint probability distributions were created by probabilistic inversion (Havelaar et al., 2008). 


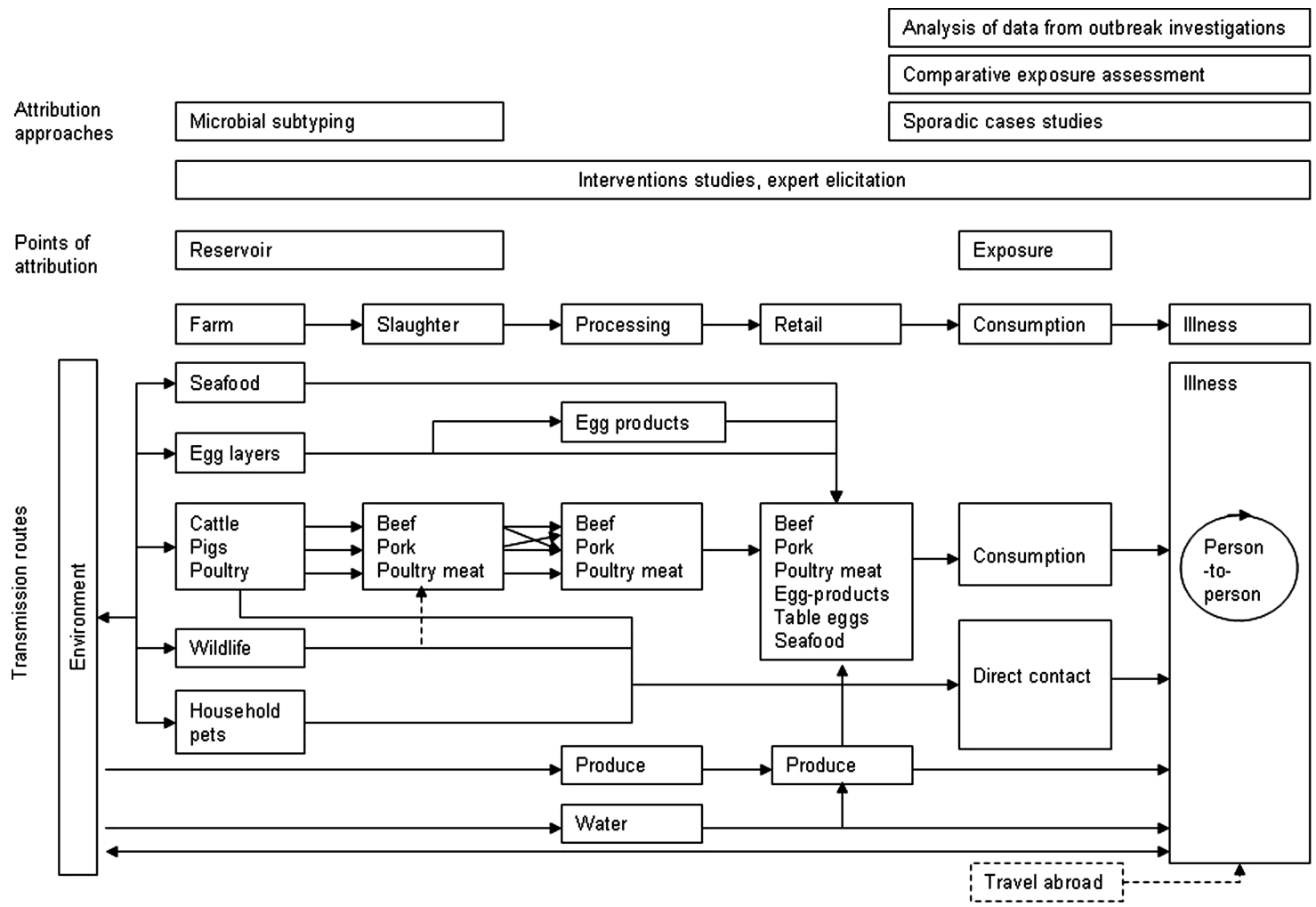

FIG. 1. Routes of transmission of zoonotic pathogens and points of human illness attribution. Travelling abroad is not considered to constitute a route of exposure by itself, as the main routes described will also apply for travelers. Still, it is common to include traveling abroad as a source on its own, which is the reason for including it in this diagram.

Although expert elicitation is useful when data are lacking, a limitation is that the conclusions are based on individual judgment, which may be misinformed or biased (Batz et al., 2004). Nevertheless, for some pathogens or diseases, expert elicitations may be the only available method for source attribution.

\section{Points of Source Attribution}

Human illness source attribution can take place at different points along the food chain, including at production, distri- bution and consumption. Source attribution at the point of production most closely represents attribution of the foodborne pathogen at the reservoir level. A reservoir is defined as an animal species or a nonanimal substance upon which the pathogen depends for its survival (Martin et al., 1987).

Because pathogens that cause foodborne disease may enter the food distribution chain at different points, the burden of illness caused by one disease attributed to specific sources may vary, depending on the point along the food chain the approach focuses on. For example, because cattle are the most important reservoir of Escherichia coli O157:H7, attribution of

Table 1. Harmonized Terminology and Methodology for Attribution of Human Illness to Specific Sources

Concept Definitions

Human illness source attribution

Source

Points of attribution

Approaches

Microbiological approaches

Epidemiological approaches

Expert elicitation approaches Intervention studies
Partitioning of the human disease burden of one or more foodborne infections to specific sources

Origin of the pathogen causing infection, including animal reservoirs and vehicles, e.g., foods and water.

Points in the food chain where human illness attribution can take place, including production, distribution and consumption

\section{Methods}

Microbial subtyping

Comparative exposure assessment

Studies of sporadic cases

Case-control studies

Cohort studies

Case-series studies

Analysis of data from outbreak investigations 
E. coli O157:H7 infections will partition more illness to cattle at the point of production (reservoir) than it will partition to beef at the point of consumption, since other foods, besides beef, may be contaminated with the pathogen.

Some of the general methods to attribute foodborne diseases to specific sources work primarily at one point in the food chain (e.g., epidemiological approaches work primarily at the point of consumption), while other general methods (e.g., expert elicitation approaches) can be more generally applied. The general method for source attribution chosen, and consequently the point of attribution, will depend on the availability of data and on the risk management question being addressed. Figure 1 presents the major transmission routes for foodborne infections, including zoonotic infections, and indicates at which point in the transmission chain the different approaches attribute human illness.

\section{Conclusion}

To identify and prioritize appropriate food safety interventions and to precisely measure the impact of interventions aimed at controlling foodborne diseases, it is crucial to attribute the human disease burden of foodborne infections to specific sources. Several general methods for human illness source attribution have been developed and are being utilized, and the usefulness of each depends on the pathogen, the specific public health questions being addressed, the data availability, and the strengths and limitations of the different methods.

The limitations of the different general methods for source attribution may be overcome by the integration of different methods. The described approaches often focus on only one point of the transmission chain (e.g., point of reservoir or point of exposure), which means that choosing only one approach for source attribution may be inadequate to answer specific risk management questions. The integration of source attribution approaches aims at improving the estimates at the same point or at different points of attribution. Examples include blending data from studies of sporadic infections and analysis of data from outbreak investigations (integration at the same point of attribution), blending of case-control studies and the microbial subtyping approach, and integration of the microbial subtyping approach and the comparative exposure assessment approach (integration of different points of attribution).

Like epidemiological approaches in general, source attribution approaches describe an association between the outcome (the burden of a given disease) and the specific sources or exposures. Source attribution methods attempt to attribute the burden of disease at the population level, and do not describe causation of disease at the individual level.

Human illness source attribution is increasingly used to partition human illness to the most important sources and as such support risk management strategies. In this paper, we discussed the available source attribution approaches, and introduced nomenclature that should contribute to the harmonization of concepts, definitions, and methods. Table 1 summarizes our proposal for such harmonization. We conclude by encouraging other scientists that apply human illness source attribution to clearly address and define as a minimum: 1) the sources considered, 2) the point(s) of attribution addressed and 3) the attribution approach(es) chosen, because this is crucial for the sharing of knowledge between research groups and the comparison of results among the scientific community.

\section{Acknowledgments}

Med-Vet-Net Work Package 28 was established to focus on the development and comparison of human illness attribution methods. Med-Vet-Net is a European Union "Network of Excellence" of 16 institutions in 10 countries, established to facilitate research for prevention and control of zoonoses and foodborne diseases. The kick-off meeting of the work-package joined members of the partner institutes and participants from the Centers for Disease Control and Prevention and the United States Department of Agriculture Food Safety and Inspection Service, and focused on the harmonization of terminology on attribution. A thorough discussion achieved agreement on important concepts, which have been presented here. More information about Med-Vet-Net and the work package can be found at http://www.medvetnet.org/cms/.

\section{Disclosure Statement}

No competing financial interests exist.

\section{References}

Ackers ML, Puhr ND, Tauxe RV, et al. Laboratory-based surveillance of Salmonella serotype Typhi infections in the United States. JAMA 2000;283:2668-2673.

Adak GK, Meakins SM, Yip H, et al. Disease risks from foods, England and Wales, 1996-2000. Emerg Infect Dis 2005;11:365372.

Batz MB, Doyle MP, Morris JG, et al. Attributing illness to food. Emerg Infect Dis 2005;11:993-999.

Clayton D and Hills M. Statistical Models in Epidemiology. Oxford: Oxford University Press, 1993.

de Wit M, Koopmans MPG, Kortbeek LM, et al. Gastroenteritis in sentinel general practices, the Netherlands. Emerg Infect Dis 2002;1:82-91.

Didelot X, Falush D. Inference of bacterial microevolution using multilocus sequence data. Genetics 2007;175:1251-1266.

Dingle KE, Colles FM, Ure R, et al. Molecular characterization of Campylobacter jejuni clones: a rational basis for epidemiological investigations. Emerg Infect Dis 2002;8:949-955.

Dingle KE, Colles FM, Wareing DRA, et al. Multilocus sequence typing system for Campylobacter jejuni. J Clin Microbiol 2001;39:14-23.

[EFSA] European Food Safety Authority. The Community Summary Report on Trends and Sources of Zoonoses, Zoonotic Agents, Antimicrobial Resistance and Foodborne Outbreaks in The European Union in 2005. The EFSA Journal 2006;94:288.

Engberg J. Contributions to the epidemiology of Campylobacter infections, a review of clinical and microbiological studies. København: Statens Serum Institute, Lægeforeningens Forlag, 2006.

Evers EG, Van Der Fels-Klerx HJ, Nauta MJ, et al. Campylobacter source attribution by exposure assessment. Int J Risk Assess Manag 2008;8:174-190.

[FAO/WHO] Food and Agricultural Organization/World Health Organization. The use of microbiological risk assessment outputs to develop practical risk management strategies: metrics to improve food safety. Available from http://www .who.int/foodsafety/micro/jemra/meetings/2005/en/. 2006.

French N. Campylobacter: diversity, host specificity and population biology. Zoonoses and Public Health, 54: Abstract 0028. 
14th International Workshop on Campylobacter, Helicobacter and Related Organisms, Rotterdam, The Netherlands, September 2-5, 2007.

Gilks WR, Richardson S, and Spiegelhalter DJ. Markov Chain Monte Carlo in Practice. London: Chapman and Hall, 1996.

Hald T, Vose D, Wegener HC, et al. A Bayesian approach to quantify the contribution of animal-food sources to human salmonellosis. Risk Anal. 2004;24:251-265.

Havelaar AH, Galindo AV, Kurowicka D, et al. Attribution of foodborne pathogens using structured expert elicitation. Foodborne Pathog Dis 2008;5:649-659.

Henson S. Estimating the incidence of food-borne Salmonella and the effectiveness of alternative control measures using the Delphi method. Int J Food Microbiol 1997;35:195-204.

Hoffmann S, Fischbeck P, Krupnick A, et al. Using expert elicitation to link foodborne illnesses in the United States to foods. J Food Prot 2007;70:1220-1229.

Lake R, Cressey P, and Gallagher E. Expert opinion as a method for estimating attribution of foodborne infection sources. In: Abstracts of the Priority Setting of Foodborne and Zoonotic pathogens, Berlin, Germany, July 19-21, 2006.

Martin SW, Meek AH, and Willeberg P. Veterinary Epidemiology: Principles and Methods. Ames: Iowa State University Press, 1987.

McBride G, Meleason M, Skelly C, et al. Preliminary relative risk assessment for Campylobacter exposure in New Zealand: 1. National model for four potential human exposure routes; 2. Farm environmental model. NIWA Client Report: HAM2005094, 2005.

McCarthy ND, Colles FM, Dingle KE, et al. Host-associated genetic import in Campylobacter jejuni. Emerg Infect Dis 2007;13: 267-272.

Mead S, Slutsker L, Dietz V, et al. Food-related illness and death in the United States. Emerg Infect Dis 1999;5:607-625.

Mølbak K, Neimann J. Risk factors for sporadic infection with Salmonella Enteritidis, Denmark 1997-1999. Am J Epidemiol 2002;156:654-661.

Olsen JE, Brown DJ, Skov MN, et al. Bacterial typing methods suitable for epidemiological analysis. Applications in investigations of salmonellosis among livestock. Vet Q 1993;15:125135.

Olsen SJ, MacKinon LC, Gouldin JS, et al. Surveillance for foodborne disease outbreaks-United States, 1993-1997. MMWR CDC Surveill Summ 2000;49:1-51.

Reingold AL. Outbreak investigations-a Perspective. Emerg Infect Dis 1998;4:21-27.

Sarwari AR, Magder LS, Levine P, et al. Serotype distribution of Salmonella isolates from food animals after slaughter differs from that of isolates found in humans. J Infect Dis 2001;183: 1295-1299.

Shapiro RL, Altekruse S, Hutwagner L, et al. The role of Golf Coast oysters harvested in warmer months in Vibrio vulnificus infections in the United States, 1988-1996. J Infect Dis 1998; 178:752-759.

Sobel J, Tucker N, Sulka A, et al. Foodborne botulism in the United States, 1990-2000. Emerg Infect Dis 2004;10:16061611.

Stafford RJ, Schluter PJ, Wilson AJ, et al. Population-attributable risk estimates for risk factors associated with Campylobacter infection, Australia. Emerg Infect Dis 2008;14:895-901.

Stern NJ, Hiett KL, Alfredsson GA, et al. Campylobacter spp. in Icelandic poultry operations and human disease. Epidemiol Infect 2003;130:23-32.

Tenover FC, Arbeit RD, and Goering RV. How to select and interpret molecular strain typing methods for epidemiological studies of bacterial infections: a review for healthcare epidemiologists. Infect Control Hosp Epidemiol 1997;18:426-439.

Van Duynhoven YTHP, De Wit MAS, Kortbeek LM, et al. Voedselinfecties in Nederland. Ned Tijdschr Med Microbiol 2002;10:79-83.

Van Pelt W, Van De Giessen AW, Van Leeuwen WJ, et al. Oorsprong, omvang en kosten van humane salmonellose. Deel 1. Oorsprong van human salmonellose met betrekking tot varken, rund, kip, ei en overige bronnen. Infectiezikten Bulletin 1999;10:240-243.

Vellinga A and Van Loock F. The dioxin crisis as an experiment to determine poultry-related Campylobacter enteritis. Emerg Infect Dis 2002;8:19-22.

Voetsch AC, Kennedy MH, Keene WE, et al. Risk factors for sporadic Shiga toxin-producing Escherichia coli O157 infections in FoodNet sites, 1999-2000. Epidemiol Infect 2007;135:9931000.

Wegener HC, Hald T, Lo Fo Wong DM, et al. Salmonella control programs in Denmark. Emerg Infect Dis 2003;9:774-780.

Wheeler JG, Sethi D, Cowden JM, et al. Study of infectious intestinal disease in England: rates in the community, presenting to general practice, and reported to national surveillance. Br Med J 1999;318:1046-1050.

[WHO] World Health Organization. The World Health Report 2005-making every mother and child count. Geneva, Switzerland: WHO, 2005.

Wingstrand A, Neimann J, Engberg J, et al. Fresh chicken as main risk factor for campylobacteriosis, Denmark. Emerg Infect Dis 2006;12:280-285.

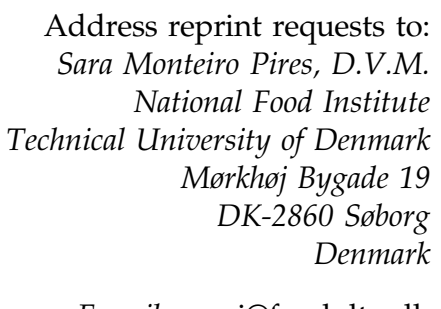

E-mail: smpi@food.dtu.dk 